\title{
Écrire la guerre sans fin : le roman Syngué Sabour. Pierre de patience d'Atiq Rahimi
}

\author{
Gabriella KÖRÖMI \\ École Supérieure Károly Eszterházy \\ Hongrie
}

\begin{abstract}
Résumé : Bien que Syngué Sabour. Pierre de patience d'Atiq Rahimi ne soit pas un récit de guerre traditionnel, c'est la guerre qui est l'élément constitutif du roman. L'histoire se passe, selon toute vraisemblance, en Afghanistan, dans ce pays où la guerre est devenue continue et où elle fait partie intégrante du quotidien des Afghans depuis plusieurs générations. Comment représenter la brutalité d'une guerre sans fin sans tomber dans le sensationnel ou le pathétique? Quels procédés stylistiques et poétiques Rahimi utilise-t-il pour raconter la violence de la guerre? Si les mots sont déjà impuissants, à quelles stratégies extra-langagières l'écrivain peut-il recourir pour dire l'indicible? Ce sont les questions auxquelles la présente étude essaie de donner des réponses.
\end{abstract}

\begin{abstract}
Atiq Rahimi's Syngué Sabour is not a classical war novel, in spite of the fact that war is the organizing theme of the novel. The plot of the novel may be set in Afghanistan, a country where war has been a permanent and integral part of the people's everyday life for generations. How is it possible to describe a never ending, dreadful war without making the writing sensationalist or pathetic? What kinds of language tools and stylistic devices are used by Rahimi to describe the violence of war? When words are not adequate for the depiction of the violence of war, what extralinguistic strategies can the writer employ? These are the research questions the article explores.
\end{abstract}

Mots-clés: guerre continue, violence de guerre, procédés stylistiques, stratégies extra-langagières.

Keywords : permanent war, the violence of war, stylistic devices, extralinguistic strategies.

Dans l'abondante production littéraire qu'elle a suscitée depuis l'Antiquité jusqu'à nos jours, la guerre est indissolublement liée au thème de la violence. Raconter cette dernière est une mission extrêmement difficile, du fait de la complexité des événements, des actes et des facteurs psychologiques qui la constituent. Pourtant, écrire la violence de guerre est bel et bien un défi qu'ont relevé de nombreux romanciers et poètes en essayant de dire l'indicible. Défi de taille, surtout quand il s'agit de la mise en scène d'une guerre qui n'en finit pas. Le terme désigne une guerre qui, par son caractère continu, devient indissociable de l'ordre politique et social d'un pays, qui, pour ainsi dire, cesse d'être un événement anormal dans la vie d'un peuple. Les questions qui ne manquent pas de se poser sont les suivantes : Comment écrire une guerre qui, au lieu d'être inscrite dans la mémoire collective d'un peuple, passe pour la seule expérience quotidienne qu'il connaisse? Dans la fiction littéraire, est-il possible de représenter différemment la violence d'une guerre sans fin que celle d'une guerre traditionnelle? Y-a-t-il vraiment des différences entre ces deux types de guerre? Si oui, comment les présenter sans tomber dans le sensationnel ou le pathétique ? Comment y sensibiliser les lecteurs? 
Il peut sembler étrange à première vue d'examiner le roman Syngué Sabour. Pierre de patience ${ }^{1}$ d'Atiq Rahimi ${ }^{2}$ sous l'angle de la thématique de la guerre sans fin. D'une part, ce n'est pas un récit de guerre proprement dit, puisque le thème principal du roman est la prise de conscience d'une femme grâce à la puissance libératrice de la parole. D’autre part, bien que la dédicace ${ }^{3}$ place d'emblée le roman sous le signe de la brutalité, celle de la guerre n'est qu'un seul maillon dans la longue chaîne de violences de toutes sortes physiques, morales, sexuelles, verbales - présentes dans le roman. Et pourtant, une lecture plus approfondie révèle que la guerre est un élément constitutif dans notre corpus, comme elle l'est dans la vie des Afghans. Le roman, même s'il ne met pas la guerre au premier plan, y est profondément ancré : celle-là sous-tend sa structure, en détermine le cadre spatio-temporel, tisse la vie des personnages principaux et secondaires.

Avant d'entamer l'essentiel de la problématique, il ne serait pas inutile de rappeler le contenu du roman. Syngué Sabour raconte l'histoire d'une femme qui veille son mari grièvement blessé, tombé dans le coma. Un mari qu'elle connaît à peine et qui ne la connaît pas du tout. Abandonnée de tous dans une ville ravagée par la guerre, la femme - pour la première fois dans sa vie prend la parole pour raconter à son mari inconscient les humiliations qu'elle a subies durant sa vie, pour lui confesser ses secrets et ses péchés. Durant ce long monologue douloureux, la femme réalise que son mari est sa syngué sabour à elle, pierre magique des contes persans, à laquelle l'on confie tout ce qu'on a sur le cœur. Le jour où la pierre de patience aura absorbé tous les secrets et tous les malheurs, elle finira par éclater et celui qui lui parle sera délivré de toutes ses souffrances. C'est ce qui arrive à l'héroïne à la fin de l'histoire également...

Syngué Sabour met en valeur le trait caractéristique de l'écriture de Rahimi : le romancier semble économiser les mots. Dans sa "langue décharnée », pour reprendre l'heureuse formule de Bernard Quiriny (2008, 25), aucun élément n'est superflu, bien au contraire, chaque mot a une importance particulière. Là où la parole semble insuffisante ou impuissante, Rahimi recourt à d'autres moyens qui sont capables de dépasser les limites du langage : silence, non-dit, diverses stratégies formelles. Dans la présente étude, nous montrerons, à travers quelques exemples concrets, l'usage particulier que le romancier réserve à ses mots, les procédés stylistiques et les stratégies de diverses sortes, pour dire ce que les lecteurs européens ne connaissent pas, heureusement: la guerre sans fin.

\footnotetext{
${ }^{1}$ Rahimi, Atiq. Syngué sabour. Pierre de patience. Paris : P.O.L., 2008. Dans le présent article, les citations tirées du roman seront suivies du titre abrévié $(S S)$ et du numéro de la page.

${ }^{2}$ Né en 1962 à Kaboul, Atiq Rahimi a fui son pays natal en 1984. Il s'est réfugié en France où il a demandé et obtenu l'asile politique. Il y vit depuis 1984. En 2008, il s'est vu décerner le prix Goncourt pour Syngué Sabour qui est son premier roman écrit directement en français.

${ }_{3}^{3}$ Ce récit, écrit à la mémoire de N.A. - poétesse afghane sauvagement assassinée par son mari-, est dédié à M.D.» $(S S, 9)$.
} 
La phrase qui introduit le roman, telle une didascalie, situe l'histoire dans un espace géographique réel : "Quelque part en Afghanistan ou ailleurs » (SS, $13)^{4}$. Dans le roman, c'est le seul indice qui nous autorise à situer l'histoire dans le pays natal du romancier ${ }^{5}$. Mais le mot «ailleurs» nous invite à une autre lecture possible, de portée plus universelle. La valeur généralisante du roman n'est que renforcée par l'absence de toutes indications précises ou identifiables, y compris le nom des personnages: les époux sont désignés simplement par «la femme» et "l'homme». La suggestion d'une portée généralisante vise à sensibiliser les lecteurs à la violence qui sera mise en scène dans le roman: par cette phrase éloquente, l'écrivain dit par avance que ce qu'il raconte peut arriver dans d'autres pays également, car, en effet, toutes les guerres se ressemblent.

Le roman Syngué Sabour est un huis clos, dans la mesure où toute l'histoire se passe dans la pénombre d'une chambre dont la femme sort rarement, mais que le narrateur ne quitte jamais. Son horizon est limité à celui de la chambre : il ne raconte que ce qu'il voit, entend, perçoit dans cet espace clos. Par conséquent, le lecteur ne reçoit que quelques rares informations de ce qui se déroule en dehors des murs de la chambre, dans la ville ravagée par les combats incessants. Cette technique narrative rend impossible toute description de combat, toute vision globale sur la guerre, par contre elle privilégie des faits isolés et traumatisants qui marquent de leur empreinte la vie du personnage. En y recourant, l'écrivain rétrécit notre champ de perception, met la guerre à la hauteur de l'homme.

Le trait le plus frappant de la guerre interminable dont l'image se dessine peu à peu sur les pages du roman, c'est qu'elle est perçue par la femme comme le cadre ordinaire de la vie. La violence est banalisée à tel point que les gens s'y sont habitués, surtout les enfants qui, nés dans la guerre, ne connaissent pas une vie normale.

Afin de surmonter l'impuissance du langage à dire le paradoxe de l'anormalité devenue normale, Atiq Rahimi fait souvent appel à l'ouïe. Bruit de

\footnotetext{
4 Rappelons que l'Afghanistan avait été occupé par l'URSS en 1979. Après le retrait des troupes de l'Armée rouge, le pays a sombré dans la guerre civile qui n'a pris fin qu'en 2001. Les bombardements de Kaboul par les Américains, provoqués par les attentats du 11 septembre, avaient pour but d'appuyer l'action de l'Alliance du Nord qui a tenté de renverser le régime des talibans, venus au pouvoir en 1996. C'est à ce moment-là qu'une nouvelle guerre a commencé, opposant ces derniers à la coalition menée par les États-Unis. Le roman Syngué Sabour a été publié en 2008, c'était donc la vingt-neuvième année de la guerre interminable que l'Afghanistan a vécue au tournant du siècle.

${ }^{5}$ Bernadette Rey Mimoso-Ruiz en a relevé un autre, suggéré par les couleurs dominantes du roman: «De manière plus allusive, la combinatoire des couleurs : le noir des nuits et du chapelet, le rouge pourpre de la robe ou l'écarlate du sang, le vert du rideau et le jaune solaire, éveille, dans le non-dit, l'évocation du drapeau afghan et élève le roman au rendu poétique d'une situation politique. » $(2009,101)$.

${ }^{6}$ Bernadette Rey Mimoso-Ruiz (2009, 91) y voit l'influence de l'écriture de Marguerite Duras, à qui Rahimi voue une admiration affichée et à qui, selon toute vraisemblance, Syngué Sabour a été dédié.
} 
bottes, appel aux armes, tirs, explosions, roulement des chars, tous ces bruits violents exprimant la menace permanente font partie intégrante du paysage sonore du récit, auxquels les personnages semblent ne plus faire attention : «Loin, quelque part dans la ville, l'explosion d'une bombe. Violente, elle détruit peut-être quelques maisons, quelques rêves. On riposte. Les répliques lacèrent le silence pesant de midi, font vibrer les vitres, mais ne réveillent pas les enfants. Elles immobilisent pour un instant - juste deux grains de chapelet - les épaules de la femme. » $(S S, 18)$. Il paraît que les bruits de combat ont une certaine cadence quotidienne : ils retentissent à la nuit qui tombe et se taisent au lever du soleil. Dans le roman, ce sont les bruits s'écartant de cette régularité, ainsi que les nuits silencieuses qui sortent de l'ordinaire.

Nous pouvons relever un contraste frappant entre le paysage sonore intérieur et extérieur du roman, et cela dès les premières pages. Comme le remarque Rey Mimoso-Ruiz, cette rupture a un sens symbolique: «L'alternance du silence intérieur et des sons extérieurs qui déchirent l'espace domestique signe implicitement la double violence de l'Afghanistan, silencieuse et martiale. » (2009, 95). Par «violence silencieuse », Rey MimosoRuiz entend certainement tout ce dont sont victimes les femmes afghanes au quotidien, indépendamment de la guerre.

La guerre sans fin étant ce qu'elle est, le thème de la mort, dont la représentation est problématique de divers points de vue, doit être nécessairement abordé par l'écrivain : dans les conflits armés, l'anéantissement définitif des êtres humains n'est qu'un moyen justifié par la fin. Impensable pour l'homme, «la mort est indescriptible, inénarrable» (Picard 1995, 27). Confronté à l'impuissance des mots devant cet indescriptible, Rahimi recourt de nouveau au sens auditif : "Au dehors, quelque part, pas très loin, quelqu'un tire une balle. Un autre, plus proche, riposte. Le premier tire une deuxième balle. L'autre ne répond plus. » (SS, 27-28).

Ce qui frappe l'oreille du narrateur, c'est la rupture du rythme habituel des tirs. Tandis que, dans un cadre banal, le silence se situe en deçà de l'attention, dans ce contexte de guerre, les lecteurs doivent y prêter attention, ce qui le rend dès lors audible, porteur de sens. Dans l'exemple choisi par nous, le silence, suggéré par la riposte qui ne vient pas, devient la métonymie de la mort. Ce silence parlant est doublé par le blanc typographique, considéré dans la poésie moderne comme l'équivalent visuel du silence. En séparant l'extrait cité du paragraphe suivant - dans lequel la femme reprend le fil de son récit interrompu pour un seul instant à cause du retentissement des tirs -, ce blanc met quasiment entre parenthèses la mort. Par cette stratégie formelle, Rahimi souligne le fait brutalement banal qu'après la mort survenue, la vie continue. De plus, le blanc de la fin du passage permet aux lecteurs de s'arrêter un instant pour réfléchir à ce que le texte leur dit. Nous devons y ajouter que dans Syngué Sabour, le romancier use abondamment du blanc typographique.

Cette rencontre inhabituelle des silences auditif et visuel, fondée sur le dehors insaisissable du langage, en dit plus long sur la mort que des dizaines de mots. Le passage sommaire, au lieu de décrire, ne fait qu'annoncer la mort 
subite d'un combattant anonyme. Sa brièveté fait naître dans l'esprit des lecteurs l'idée de la futilité de cette mort, mais aussi celle de l'indifférence avec laquelle on la conçoit. Cette indifférence n'est dite que beaucoup plus tard, quand le lecteur réalise que dans cette ville «sourde et aveugle » $(S S, 76)$, les cadavres gisant dans les rues ne sont pas enterrés, probablement parce qu'il y en a trop.

Cette nuit, on ne tire pas.

Sous la lumière fade et froide de la lune, les chiens errants aboient dans tous les coins de la ville. Jusqu'à l'aurore.

Ils ont faim.

Ce soir il n'y a pas de cadavre. (SS, 127)

Face à la réalité inhumaine, l'écrivain se sent dépourvu de mots et recourt au non-dit, qui est considéré comme une autre forme du silence. Entre le silence dû à l'absence des tirs et la faim des chiens, il n'y a qu'une causalité indicible. Pour que les lecteurs parviennent à la comprendre, ils ont besoin du support de la phrase négative très laconique, laquelle est suivie d'un blanc typographique expressif, lui aussi.

La mort présentée ci-dessus se produit quelque part dans la ville et n'a aucun lien explicite avec l'héroïne. Mais le roman évoque une autre mort, beaucoup plus sanglante, qui frappe ses voisins. Après les combats de nuit, ayant peur des représailles, la femme se cache avec ses deux filles dans le sous-sol. Elle dort d'un sommeil si profond, qu'elle n'entend rien de ce qui se passe près de chez elle: les soldats de l'autre camp - lequel n'est nullement identifié - pénètrent dans la maison voisine. L'horrible massacre est présenté du point de vue du narrateur, qui n'en entend rien, lui non plus. À travers les rideaux, il n'aperçoit que le va-et-vient de l'ombre de la vieille voisine; à travers le mur, il n'entend que des bruits apparemment banals. Le passage en question est parsemé de verbes exprimant des mouvements et des sons, ou bien leur absence :

Elle tousse et marmonne un nom inaudible. Elle tousse. Elle attend. Vainement. Elle bouge, s'éloigne, marmonne de nouveau le nom, et tousse. Sans réponse aucune. Elle appelle, elle tousse. Elle n'attend plus. Elle ne marmonne plus. Elle chantonne quelque chose. Des noms, peut-être. Et s'en va. Loin. Puis revient. On l'entend toujours chantonner, malgré le bruit de la rue. Le bruit des bottes. Les bottes de ceux qui sont munis d'armes. (SS, 48).

Bien que rien ne soit dit explicitement, les procédés stylistiques utilisés par le romancier sont significatifs à plusieurs égards : le rythme saccadé des phrases courtes, souvent verbales, allié au bruit pesant des bottes, traduit une écriture agressive qui semble se décomposer sous nos yeux. Il peut paraittre paradoxal, mais la puissance expressive du texte vient de son morcellement. C'est justement le style haché qui suggère un acte extrêmement violent, soigneusement passé sous silence.

Celui-ci ne sera révélé que le lendemain, quand la femme veut voir le mari et le fils de sa voisine. La femme pose quelques questions, auxquelles elle 
ne reçoit pas de réponse. Plus exactement, la vieille y répond en racontant un conte un peu énigmatique sur un roi, et en chantant quelques mesures d'un air, dans lequel il s'agit également d'un roi $^{7}$. La vieille finit par éclater en sanglots et invite la femme à voir ses hommes à elle, lesquels, comme elle dit, "tiennent leur tête sous le bras » $(S S, 56)$. Le narrateur saisit encore le bruit des pas des deux femmes qui s'éloignent, ensuite il ne perçoit qu'un silence profond: "Soudain, un hurlement, celui de la femme. Horrifiée. Horrifiante. Ses pas dégringolent sur les dalles, trébuchent sur les ruines, traversent le jardin et rentrent dans la maison. Elle crie, toujours. Elle vomit. Elle pleure. Elle court dans la maison. Comme une folle. » $(S S, 56)$.

Contrairement à la présentation de la mort du combattant, dans ce cas-là, ce sont les divers moyens langagiers qui ont le rôle primordial dans l'écriture du meurtre. Nous pouvons constater que le romancier a parsemé le texte du conte, ainsi que les paroles de la chanson, des mots et des expressions qui semblent être prémonitoires. À partir de ces mots-clés (comme par exemple «beau à mourir », " danse des morts », " couper la tête », "verser de l'huile brûlante sur le corps », "tenir la tête sous le bras »), pareillement aux pièces d'un puzzle à assembler, le lecteur reconstitue pas à pas ce qui s'est passé : les combattants ont massacré le mari et le fils de la vieille, qui, restée auprès des cadavres ensanglantés des siens, est devenue folle. Le lecteur réussit à combler les lacunes du récit du meurtre non-dit grâce aux brèves phrases violentes, décrivant les réactions psychiques et physiques des deux femmes choquées par la vue des hommes décapités.

Sous l'angle de cette révélation, le passage précédent revêtira une signification nouvelle. Les phrases fragmentaires un peu inquiétantes, mais innocentes à la première lecture, se transforment en signes évidents de l'aliénation. Le lecteur n'y voit plus les gestes répétitifs d'une femme âgée, mais les symptômes d'un trouble obsessionnel, provoqué par le trauma vécu.

Ces passages sont importants du point de vue de l'intertextualité également. L'insertion des contes, des airs ou des comptines dans le texte est un procédé fréquemment utilisé par le romancier.

En Orient, où les contes ont une tradition ancestrale, leur raison d'être réside dans leur rôle médiatif : ils servent non seulement à (re)vivre la réalité, mais à l'interpréter également. Cette fonction du conte revêt une importance particulière dans Syngué Sabour (comment ne pas penser au mythe raconté par le beau-père de la femme, donnant son titre au roman?), où les contes intercalés sont destinés soit à dire tout ce que les simples mots ne peuvent pas dire - secrets, tabous, brutalités, mort -, soit à faire réfléchir les lecteurs, ce qui prolonge en quelque sorte le récit au-delà de la fiction. Par l'insertion des contes dans son roman, Atiq Rahimi rend hommage à la culture orientale, sa culture natale. Quand on pose une question à un sage persan, au lieu de

\footnotetext{
7 Il est notoire que dans de nombreuses cultures, la figure du roi représente le symbole de la domination et de la force. Cf. Cazenave, Michel. Encyclopédie des symboles. Paris : Librairie Générale Française, 1996, 580-584.
} 
donner une réponse concrète ou précise, il raconte un conte n'ayant ni fin, ni morale. "Cela vient, je crois, de la culture indienne. Dans la culture indienne, le sens n'est pas au bout d'une histoire. Le sens traverse l'histoire; ce que, ici, plus tard, dans les années 1960, Barthes a étudié. Le sens est parsemé. Le sens étoile le texte. » - a dit Rahimi dans une interview (Profizi 2013, en ligne).

Les propos cités mettent en relief le double enracinement du roman Syngué Sabour: l'écriture minimaliste et la tradition ancienne des contes. De cet heureux mariage des techniques narratives occidentale et orientale résulte un texte particulièrement dense, pareil à un scénario qui est fondé sur les faits visuels et auditifs. Ce type d'écriture a pour résultat un style sobre: ton impersonnel, voire froid, phrases brèves, dépouillées de tout élément superflu, descriptions concises.

Une autre remarque s'impose à cet endroit. À la lumière de l'affirmation d'Atiq Rahimi, nous devons compléter ce que nous avons dit ci-dessus du blanc typographique. Il paraît que son utilisation est en rapport étroit avec le concept du texte étoilé, brisé en des unités de signification indépendantes. Par ce moyen d'ordre formel, Rahimi réussit à mettre en valeur les passages ayant un sens particulièrement important du point de vue diégétique ou médiatif.

Nous avons constaté plus haut que le blanc typographique, porteur de sens, est un outil dont on use dans la poésie moderne. Mais la poésie se manifeste dans le texte d'une autre façon également. Il existe plusieurs passages qui s'apparentent à la prose poétique. À titre d'exemple, citons un passage qui décrit le décor morne de la ville en état de guerre :

Le soleil se couche.

Les armes se réveillent.

Ce soir encore on détruit.

Ce soir encore on tue.

Le matin.

Il pleut.

Il pleut sur la ville et ses ruines.

Il pleut sur les corps et leurs plaies. (SS, 70-71).

C'est un texte soigneusement construit dont le lexique est relativement restreint, mais qui en dit long grâce aux répétitions et aux antonymes. Faute de rimes, il est rythmé par les allitérations (soleil se couche, ce soir), les oppositions (soir/matin, ville/ruine, corps/plaies) et les anaphores (ce soir, il pleut). Les phrases portant en elles une métrique cadencée, sont très suggestives. L'effet musical, ainsi que la souplesse de la prose font un contraste brutal avec le caractère interminable de la guerre, exprimé par le mot « encore ». Pour conclure, nous pouvons constater que, dans le roman, c'est la prose poétique qui redonne au vocabulaire simpliste de guerre son pouvoir émotionnel et sensationnel.

Il nous reste à examiner le cas de la violence sexuelle, à laquelle les femmes sont confrontées dans tout conflit armé. Cet acte est souvent considéré comme une arme de guerre parmi tant d'autres. Le roman de Rahimi ne fait aucune allusion à cette interprétation de la violence sexuelle. 
L'écriture du viol des femmes est un sujet délicat dans une culture où les femmes peuvent être contraintes à des relations sexuelles dans la vie quotidienne, en temps de paix également. Sous cet angle, il n'est pas surprenant que la femme soit violée par un combattant, appartenant probablement au même camp que son mari. Quand des hommes armés pénètrent dans la chambre, la femme, qui sait bien que la violence est inscrite au « code du guerrier» des hommes afghans, prétend être pute. Comme elle l'explique plus tard à son mari inconscient: "J'étais obligée de lui dire ça, sinon, il m'aurait violée. [...] Pour les hommes comme lui, baiser, violer une pute, ce n'est pas un exploit. [...] Violer une pute, ce n'est pas un viol. Mais voler la virginité d'une fille, violer l'honneur d'une femme! Voilà votre credo!» $(S S, 89)$.

Remarquons, entre parenthèses, que la femme compte son mari parmi ces brutes à la recherche de butin (objets de valeur ou femmes), ce qui en dit long sur son mariage, sur sa sexualité.

Les hommes croient le mensonge et s'enfuient de la maison impie. Mais un adolescent bégayant, victime des tortures de ses propres camarades, revient au lendemain. La femme essaie de s'expliquer, mais en vain, il la contraint à coucher avec lui.

Le narrateur raconte l'acte de viol d'un style objectif, très sec. La description a un rythme particulier dû à l'alternance des phrases ayant pour sujet les pronoms «il/lui» ou «elle». Cette alternance permet au narrateur d'écrire successivement tous les gestes des deux personnages. Ceux de l'homme sont brusques, violents, tandis que la femme est pétrifiée.

Dans le passage en question, les lecteurs peuvent révéler plusieurs signes qui font penser à un trouble post-traumatique chez la femme : engourdissement, pleurs, cri. Mais rien n'est dit, rien n'est détaillé, ici non plus. Nous n'avons que les faits nus: "La femme est toujours assise à la même place. Elle reste là pendant longtemps. Sans regarder vers le rideau vert. Ses yeux s'embuent de larmes. Son corps se replie. Elle prend ses genoux entre ses bras, enfouit sa tête, et crie. Un seul cri, déchirant. » (SS, 108).

La focalisation externe du passage ne peut pas rendre ce que la femme éprouve ou pense exactement, le narrateur le déduit des gestes qu'elle fait. Puisque l'écriture est réduite aux apparences extérieures de la réalité perçue, le narrateur semble incertain de ce qu'il voit: «Le froid ou l'émotion, les larmes ou la terreur saccadent son souffle. Elle tremble. » $(S S, 108)$.

La technique de la focalisation externe fait appel à la participation active des lecteurs. Il faut qu'ils ramassent tous les indices dont le texte est parsemé, il faut qu'ils y réfléchissent, sinon ils peuvent se méprendre sur ce qu'ils ont lu. Cette stratégie, invitant les lecteurs à relire et repenser le texte, est inhérente à l'écriture fragmentaire du romancier. Plus le style est minimaliste, plus l'association d'idées, évoquée par les diverses stratégies, devient indispensable à la compréhension.

En guise de conclusion, nous pouvons constater que dans Syngué Sabour, Rahimi, au lieu de décrire la guerre sans fin, nous dit l'histoire d'une femme 
qui la vit, les répercussions que les violences subies ont sur son destin, et cela avec une extrême économie des mots. Ce qui assure une place à part au roman dans l'abondante production littéraire de guerre, c'est la puissance poétique de la parole, soutenue par le silence pesant et le vide du blanc.

\section{Références bibliographiques}

Cazenave, Michel. Encyclopédie des symboles. Paris : Librairie Générale Française, 1996.

Picard, Michel. La littérature et la mort. Paris : PUF, 1995.

Profizi, Alexandra. "Atiq Rahimi : "En Afghanistan, l'individu n'existe pas" ». La règle $d u$ jeu. [En ligne]. Mis en ligne le 12 avril 2013. URL: http:/ /laregledujeu.org/2013/04/12/13017/atiq-rahimi-en-afghanistanlindividu-nexiste-pas/. (Consulté le 6 juin 2015).

Quiriny, Bernard. «Les raisons d'un succès, Pierre précieuse ». Le Magazine Littéraire No. 481 (2008) : 25.

Rahimi, Atiq. Syngué sabour. Pierre de patience. Paris : P.O.L., 2008.

Rey Mimoso-Ruiz, Bernadette. «Silence du cyan et cri d'écarlate. Syngué sabour. La Pierre de patience (Atiq Rahimi, 2008)». Logosphère, «Écritures du silence » N5 (2009) : 89-103. 
